Cite this: Phys. Chem. Chem. Phys., 2013,

\title{
Chemically synthesised atomically precise gold clusters deposited and activated on titania. Part II†
}

15, 14806

\author{
David P. Anderson, ${ }^{a}$ Rohul H. Adnan, ${ }^{a b}$ Jason F. Alvino, ${ }^{c}$ Oliver Shipper,$t^{c}$ \\ Baira Donoeva, ${ }^{a}$ Jan-Yves Ruzicka, ${ }^{a}$ Hassan Al Qahtani, ${ }^{d}$ Hugh H. Harris, ${ }^{c}$ \\ Bruce Cowie, ${ }^{e}$ Jade B. Aitken, ${ }^{f}$ Vladimir B. Golovko, ${ }^{\text {*a }}$ Gregory F. Metha ${ }^{\star c}$ and \\ Gunther G. Andersson*d
}

\begin{abstract}
Synchrotron XPS was used to investigate a series of chemically synthesised, atomically precise gold clusters $\mathrm{Au}_{n}\left(\mathrm{PPh}_{3}\right)_{y}(n=8,9$ and 101, $y$ depending on the cluster size) immobilized on anatase (titania) nanoparticles. Effects of post-deposition treatments were investigated by comparison of untreated samples with analogues that have been heat treated at $200{ }^{\circ} \mathrm{C}$ in $\mathrm{O}_{2}$, or in $\mathrm{O}_{2}$ followed by $\mathrm{H}_{2}$ atmosphere. XPS data shows that the phosphine ligands are oxidised upon heat treatment in $\mathrm{O}_{2}$. From the position of the $A u 4 f_{7 / 2}$ peak it can be concluded that the clusters partially agglomerate immediately upon deposition. Heating in oxygen, and subsequently in hydrogen, leads to further agglomeration of the gold clusters. It is found that the pre-treatment plays a crucial role in the removal of ligands and agglomeration of the clusters.
\end{abstract}

Received 16th June 2013,

Accepted 24th July 2013

DOI: $10.1039 / \mathrm{c} 3 \operatorname{cp} 52497 \mathrm{c}$

www.rsc.org/pccp

\section{Introduction}

Gold particles with a size reduced below 5 nanometres are known to be catalytically active. ${ }^{1-3}$ Moreover, it has been demonstrated that the onset of catalytic activity of gold often coincides with the loss of metallic properties of Au nanoparticles at around 1.5-2 $\mathrm{nm}$. This size-threshold is established for both naked clusters prepared under UHV conditions ${ }^{4}$ and chemically-synthesised $^{5,6}$ well-defined metal nanoparticles immobilized onto inert supports. Metal clusters can be deposited from the gas phase as size selected clusters ${ }^{7-15}$ or through electrospray ionisation ${ }^{6}$ or from the liquid phase when using chemically synthesised nanoparticles. ${ }^{16,17}$ The latter route offers the huge benefit of easy scale-up. Removal of ligands is often postulated as a necessary step for making the adsorption sites at the surface of the clusters catalytically active.

\footnotetext{
${ }^{a}$ The MacDiarmid Institute for Advanced Materials and Nanotechnology, and Department of Chemistry, University of Canterbury, Christchurch 8140, New Zealand. E-mail: vladimir.golovko@canterbury.ac.nz

${ }^{b}$ Chemistry Department, University of Malaya, 50603 Kuala Lumpur, Malaysia

${ }^{c}$ School of Chemistry and Physics, The University of Adelaide, Adelaide, SA 5005, Australia. E-mail: greg.metha@adelaide.edu.au

${ }^{d}$ Flinders Centre for NanoScale Science and Technology, Flinders University, Adelaide, SA 5001, Australia. E-mail: gunther.andersson@flinders.edu.au ${ }^{e}$ Australian Synchrotron, 800 Blackburn Road, Clayton Vic-3168, Australia

${ }^{f}$ School of Chemistry, The University of Sydney, Sydney 2006, Australia

$\dagger$ Electronic supplementary information (ESI) available: Fitting of the gold $\mathrm{Au}_{8}$ measurements and XANES fitting plots. See DOI: 10.1039/c3cp52497c

$\ddagger$ Current address: School of Chemistry, Bielefeld University, Bielefeld, Germany.
}

We have previously studied the properties of a series of chemically made atomically precise clusters deposited on the popular P-25 Aeroxide, a mixture of nanosized anatase and rutile, which was pre-treated with acid prior to deposition of clusters. We have shown that calcination (heat-treatment in vacuum) and washing in hot toluene results in the removal of some clusters from the P-25 surface. ${ }^{16}$ Of the clusters remaining on the titania surface, one fraction is virtually unchanged (from the untreated form), whereas another fraction shows formation of $\mathrm{Au}-\mathrm{O}$ bonds, most likely to the oxygen of the titania surface. No significant agglomeration nor complete removal of phosphine ligands from the clusters could be inferred from the observed XPS spectra. Heating of the support-immobilised clusters under vacuum at $200{ }^{\circ} \mathrm{C}$ has two effects. First, the ultra-small clusters aggregate to form slightly larger gold particles that are still protected by phosphine ligands. The average size of the aggregated clusters was estimated to be marginally smaller than that of the untreated $\mathrm{Au}_{101}$. Second, a fraction of gold clusters exhibit $\mathrm{Au}-\mathrm{O}$ bonds, most likely to the oxygen of the titania surface, which coincides with the loss of phosphine ligands and formation of oxidised phosphorous species.

Similar heat treatment has been used in other studies for fabricating catalysts based on chemically synthesised clusters and colloids. ${ }^{5,18,19}$ Zanella et al. focused on the parameters of thermal treatment that influence the gold particle size in $\mathrm{Au} / \mathrm{TiO}_{2}$ samples prepared by different methods. ${ }^{20}$ It has been previously shown that the type of chemical atmosphere during 
heat treatment of the $\mathrm{Au}-\mathrm{TiO}_{2}$ catalysts also has a pronounced effect on the evolution of particle size. Heat-treatment of ligand stabilised clusters has been performed in an oxygen rich atmosphere, which facilitates ligand removal due to their partial or even complete oxidation, ${ }^{21}$ while in other cases oxygen plasma was used for ligand removal. ${ }^{22}$ Finally, removal of protecting thiol ligands was recently achieved using strong oxidants $\left(\mathrm{KMnO}_{4}\right.$ and $\left.\mathrm{K}_{2} \mathrm{MnO}_{4}\right)$, although post-treatment calcination was still required to "burn off" residual organic species. ${ }^{23}$ Activation in an oxidative atmosphere is suggested to be more efficient compared to activation under a purely reducing $\left(\mathrm{H}_{2}\right)$ atmosphere, which failed to remove thiol ligands completely from support-immobilised chemically-made clusters even after 8 hours at $563 \mathrm{~K}^{24}$

The aim of the present work is an investigation of the influence of oxidative treatments on the size and environment of gold clusters deposited on untreated titania. Similar to our previous study, ${ }^{16}$ we apply X-ray photoelectron spectroscopy (XPS) of $\mathrm{Au}$ and $\mathrm{P}$ to analyse the chemical state and possible agglomeration of the gold clusters deposited onto titania. The $\mathrm{Au}$ peak position is influenced by the size of the clusters through the final state effect, ${ }^{25}$ which has been used by many others to relate XPS binding energy to size of a metal cluster..$^{8,9,17,26-30}$ In particular, we focus on the question of whether the employed treatments remove the ligands and if it impacts on the agglomeration of the metal cores.

\section{Experimental}

\section{A. Materials}

Anatase nanoparticles (99.8\%) with a particle size of about $10 \mathrm{~nm}$ and surface area of $c a .50 \mathrm{~m}^{2} \mathrm{~g}^{-1}$, were supplied by SkySpring Nanomaterials, Inc., USA and used for immobilisation of clusters without any pre-treatment. Chemically synthesised, atomically precise gold clusters $\mathrm{Au}_{n}\left(\mathrm{PPh}_{3}\right)_{y}(n=8,9,11$ and 101, with $y$ depending on cluster size) were prepared and purified using the same procedure reported by us and others. ${ }^{16}$ Instrument grade $\mathrm{O}_{2}\left(99.2 \%\right.$ with $<10 \mathrm{ppm} \mathrm{H}_{2} \mathrm{O}$, Southern Gas Services Ltd) and $\mathrm{H}_{2}\left(99.98 \%\right.$ with $<20$ ppm $\mathrm{H}_{2} \mathrm{O}$, BOC) gases were used for the post-treatments.

\section{B. Deposition of clusters onto support}

The clusters were deposited onto the support using the method reported by Zhu et al. ${ }^{31}$ First, the calculated amount of support was dried in the Schlenk tube under vacuum at $200{ }^{\circ} \mathrm{C}$ with stirring overnight $(12 \mathrm{~h})$. Upon cooling to room temperature the support was suspended in dichloromethane (AR grade, $50 \mathrm{~mL}$ ) using vigorous stirring (750 rpm, magnetic stirrer bar). A solution of calculated amount of metal cluster in dichloromethane $(20 \mathrm{~mL})$ was added to the suspension of titania under vigorous stirring at room temperature and the mixture was left stirring overnight $(12 \mathrm{~h})$. The mixture was then dried in vacuum at room temperature and stored under refrigeration. For the purpose of this study we focused on materials with 0.17 weight (wt)\% Au (i.e. ratio of the weight of the Au due to presence of clusters relative to the titania particles). This specific level of loading with clusters was chosen to minimise aggregation and still be able to study these materials using synchrotron XPS. In selected cases, cluster loading on anatase was reduced two-fold to $0.08 \mathrm{wt} \% \mathrm{Au}$ in order to investigate the effect of loading.

\section{Treatments of support-immobilised clusters}

In this study, we investigated two heat treatments of supportimmobilised clusters: (a) calcination under pure $\mathrm{O}_{2}$ atmosphere at $200{ }^{\circ} \mathrm{C}$ for $2 \mathrm{~h}$ and (b) calcination under pure $\mathrm{O}_{2}$ at $200{ }^{\circ} \mathrm{C}$ for $2 \mathrm{~h}$ followed by calcination in $\mathrm{H}_{2}$ at $200{ }^{\circ} \mathrm{C}$ for $2 \mathrm{~h}$. In all cases atmospheric pressure of the corresponding gas was used.

Typically, a Schlenk tube containing $c a .500 \mathrm{mg}$ of anatase with supported cluster at 0.17 or $0.08 \mathrm{wt} \% \mathrm{Au}$ and a magnetic stirrer bar was wrapped in foil to prevent exposure to light and connected to the Schlenk line which was connected to a pure $\mathrm{O}_{2}$ cylinder. The Schlenk tube was evacuated and refilled with oxygen while at room temperature. This vacuum- $\mathrm{O}_{2}$ cycle was repeated at least three times to ensure pure oxygen atmosphere. The Schlenk tube was then placed into a preheated oil bath on a hotplate-stirrer maintained at $200{ }^{\circ} \mathrm{C}$ via a direct thermocouplecontrolled feedback loop. Oxygen flow through the Schlenk line to which the Schlenk flask was attached was maintained at the rate of $c a$. 1 bubble per second. Heating at $200{ }^{\circ} \mathrm{C}$ with magnetic stirring continued for 2 hours. After this period the Schlenk tube was pulled from the oil bath and allowed to cool to room temperature.

In the case of $\mathrm{O}_{2}$ activation followed by $\mathrm{H}_{2}$ treatment, the Schlenk tube was subsequently attached to another Schlenk line connected to a $\mathrm{H}_{2}$ cylinder. A cycle of evacuation followed by filling with $\mathrm{H}_{2}$ was repeated at least three times at room temperature to ensure pure $\mathrm{H}_{2}$ atmosphere. After filling the Schlenk tube with $\mathrm{H}_{2}$ the heat-treatment was performed using pre-heated oil bath as described above. All the treated catalysts were stored in the dark at $4{ }^{\circ} \mathrm{C}$.

\section{Synchrotron XPS beamline and sample preparation}

A suspension of the support-immobilised cluster was made up in dichloromethane at a concentration of $c a .1 \mathrm{mg} \mathrm{mL}^{-1}$. A $10 \mu \mathrm{L}$ drop of each sample was deposited onto a clean $6 \times 6 \mathrm{~mm}$ silicon (Si) wafer and dried in air. Each sample was then fixed by double-sided copper tape onto a gold-plated holder; up to 6 samples were affixed onto each plate for XPS analysis.

Photoelectron spectra were recorded at the Soft X-ray Beamline at the Australian Synchrotron (AS) using a SPECS Phoibos 150 hemispherical electron analyser with the photon energy set to $690 \mathrm{eV}$. The beam was adjusted to an irradiation spot size of $\sim 600 \times 600 \mu \mathrm{m}$, providing an X-ray photon flux of approximately $10^{12}$ photons $\mathrm{mm}^{-2} \mathrm{~s}^{-1}$, conditions that we have recently shown not to induce thermal damage to samples of

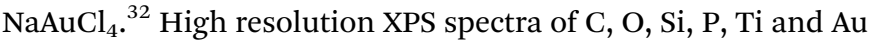
were recorded at a pass energy of $10 \mathrm{eV}$, yielding an instrumental resolution of $295 \mathrm{meV} .^{33}$ Scans were repeated several times to ensure that no photon-induced changes occurred in the samples. The stability of the X-ray energy was monitored using a bulk gold reference. 
For all XPS spectra, a Shirley background was first applied to remove the electron-scattering background and maintain the intrinsic line shape from the raw data. ${ }^{34,35}$ A pseudo-Voigt function composed of the sum of Gaussian (30\%) and Lorentzian (70\%) functions was used to fit all peaks and all peak positions were allowed to vary using nonlinear least-squares minimization. ${ }^{36}$ For the $\mathrm{Au} 4 \mathrm{f}$ doublet, splitting was fixed at $3.67 \mathrm{eV}$ while for the P 2p doublet a splitting of $0.84 \mathrm{eV}$ was used. ${ }^{37}$ All spectra where fitted with the least number of peaks allowing a variation of the FWHM, although the FWHM of a single contributing species was kept constant. For example, in the case of a gold spectrum fitted with two sets of $4 \mathrm{f}_{7 / 2}$ and $4 \mathrm{f}_{5 / 2}$ doublets, the FWHM within a single doublet was kept constant but was allowed to differ between the doublets. In order to illustrate the fitting procedure, the fits to the $\mathrm{Au}_{8}$ spectra are shown in the ESI. $\dagger$

Cross sections were calculated according to Yeh and Lindau using the photoionization cross section and the asymmetry parameter. ${ }^{38}$ The angle between the sample normal and the analyser was $10^{\circ}$ which means that the angle between the incident horizontal linearly polarised synchrotron beam and the sample surface was $45^{\circ}$.

\section{E. X-ray absorption spectroscopy (XAS)}

$\mathrm{Au} \mathrm{L}_{\mathrm{III}}$-edge X-ray absorption spectra were recorded at the Australian National Beamline Facility (ANBF, beamline 20B) at the Photon Factory, Tsukuba, Japan. Harmonic rejection was achieved by detuning a channel-cut $\mathrm{Si}[111]$ monochromator by $50 \%$. Unsupported clusters were recorded in transmission mode as pressed pellets with a 1:5 dilution in cellulose. Supported cluster samples were recorded neat in fluorescence mode with the sample suspended in a $1 \times 5 \mathrm{~mm}$ aluminium sample holder secured between Kapton tape, measured using a 36-pixel Ge-monolith fluorescence detector (Canberra/Unisys) at $90^{\circ}$ to the incident beam. All data was collected at $\sim 15 \mathrm{~K}$ using a closed-cycle He cryostat. The following energy ranges were used for X-ray absorption near-edge structure (XANES) data collection: pre-edge region from 11700 to $11900 \mathrm{eV}(10 \mathrm{eV}$ steps), XANES region from 11900 to $11960 \mathrm{eV}$ (0.5 eV steps), and post-edge region from 11960 to $13145 \mathrm{eV}\left(0.05 \AA^{-1}\right.$ steps in

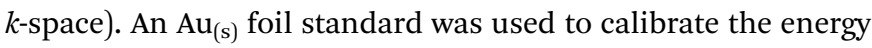
scale to the first peak of the first derivative of the $\mathrm{Au} \mathrm{L}_{\mathrm{III}}$ edge (assumed to be $11920 \mathrm{eV}$ ). Data analysis, including calibration, averaging, background subtraction of all spectra and linear regression analysis of selected XANES spectra were performed using EXAFSPAK (G. N. George, SSRL).

\section{Results and discussion}

\section{A. XPS results}

General. In the present work, the molecular clusters $\mathrm{Au}_{8}$ and $\mathrm{Au}_{9}$ contain atomically precise, monodisperse metal cores protected by phosphine ligands. ${ }^{16}$ The cluster $\mathrm{Au}_{101}$ is also used which is not atomically monodisperse but shows a narrow particle size distribution with an average size of approximately $1.5 \mathrm{~nm} .{ }^{39,40}$ The sample loadings of $0.17 \mathrm{wt} \%$ Au corresponds to approximately 1 cluster per titania particle (assuming a $10 \mathrm{~nm}$ diameter particle), or 1 cluster per about $400 \mathrm{~nm}^{2}$. The XPS results from the $0.08 \mathrm{wt} \% \mathrm{Au}$ samples are effectively the same as that observed for the $0.17 \mathrm{wt} \% \mathrm{Au}$, indicating that a two-fold reduction in the loading does not affect the behaviour of gold clusters. In particular, the two-fold decrease in cluster loading does not influence the agglomeration of the clusters, as will be discussed below. For this reason the $0.08 \mathrm{wt} \%$ Au data is not presented.

XPS spectra were recorded at the Au 4f, P 2p, Si 2p, Ti 2p, $\mathrm{C} 1 \mathrm{~s}$ and $\mathrm{O} 1 \mathrm{~s}$ regions for all samples of $\mathrm{Au}_{n}$ clusters deposited on titania nanoparticles, and also from a gold reference sample. The majority of carbon signal arises from either the triphenylphosphine ligands or adventitious hydrocarbons. In either case, we fix the $\mathrm{C} 1 \mathrm{~s}$ peak to $285 \mathrm{eV}$ and use it for calibrating the peak positions of other elements. This is justified because adventitious hydrocarbons display a very constant $\mathrm{C}$ 1s peak position that is present in all samples exposed to air (and not cleaned by sputtering prior to XPS measurements). ${ }^{41}$ The titanium signal measured is exclusively due to the titania nanoparticles and found at $459.1 \pm 0.1 \mathrm{eV}$. The silicon signal is due to the silicon wafer used as substrate and found at $98.9 \pm$ $0.2 \mathrm{eV}(\mathrm{Si})$ and $102.6 \pm 0.3 \mathrm{eV}\left(\mathrm{SiO}_{2}\right)$. The oxygen signal is mostly due to titanium dioxide $(530.4 \pm 0.2 \mathrm{eV})$ and silicon dioxide $(531.9 \pm 0.3 \mathrm{eV})$. Gold and phosphorous signals from the clusters are found in the regions 83.6-85.6 eV and 131.9$133.4 \mathrm{eV}$, respectively, and are used for quantitative analysis. Using the energy calibration described above, $\mathrm{Au} 4 \mathrm{f}_{7 / 2}$ of bulk gold is usually found at a binding energy of $84.0 \mathrm{eV}$. This value is used to determine the shift between the $\mathrm{Au} 4 \mathrm{f}_{7 / 2}$ signal of the gold clusters and the bulk gold binding energy. The variation of the silicon signals relative to the gold reference is less than $0.1 \mathrm{eV}$, and that of the titanium signal less than $0.25 \mathrm{eV}$, indicating that there is no significant charging of the samples or instability in the X-ray energy.

Untreated samples. The $\mathrm{Au}$ if spectra for the $\mathrm{Au}_{8}, \mathrm{Au}_{9}$, and $\mathrm{Au}_{101}$ clusters deposited on anatase particles and not exposed to any additional activation treatment (i.e. untreated samples) are shown in the lower traces of Fig. 1A-C. The spectra of $\mathrm{Au}_{8}$, $\mathrm{Au}_{9}$ and $\mathrm{Au}_{101}$ show two doublets while the second doublet of $\mathrm{Au}_{101}$ is negligible. For the untreated samples, the position of the $\mathrm{Au} 4 \mathrm{f}_{7 / 2}$ peak at the lower binding energy (referred to as Au-LBP) is at $83.7 \pm 0.1 \mathrm{eV}$ and that of the higher binding energy (referred to as Au-HBP) is at $85.3 \pm 0.2 \mathrm{eV}$, as shown in Fig. 2A and Table 1. The Au-LBP has a full-width-half-maximum (FWHM) of 1.0 to $1.1 \pm 0.1 \mathrm{eV}$ while the Au-HBP has a FWHM of 1.5 to $1.9 \pm 0.2 \mathrm{eV}$ (see Table 1). An example of the fitting results for $\mathrm{Au}_{8}$ is shown in the ESI. $\uparrow$ Based on the shift of the $\mathrm{Au}$ position and the increase of the FWHM, the Au-HBP has previously been assigned to non-agglomerated clusters, and the Au-LBP assigned to agglomerated clusters since its position coincides with that of bulk gold. ${ }^{16}$ For $\mathrm{Au}_{8}$ and $\mathrm{Au}_{9}$, the peak areas of Au-HBP and Au-LBP are almost the same, whereas the majority of the $\mathrm{Au}_{101}$ intensity is as Au-LBP, as seen in Fig. 2A. This is in contrast to our earlier study where the majority of signal was found as $\mathrm{Au}-\mathrm{HPB}$ for untreated $\mathrm{Au}_{8}$ and $\mathrm{Au}_{9}$ clusters deposited onto P-25 Aeroxide titania pre-treated with acid. ${ }^{16}$ 

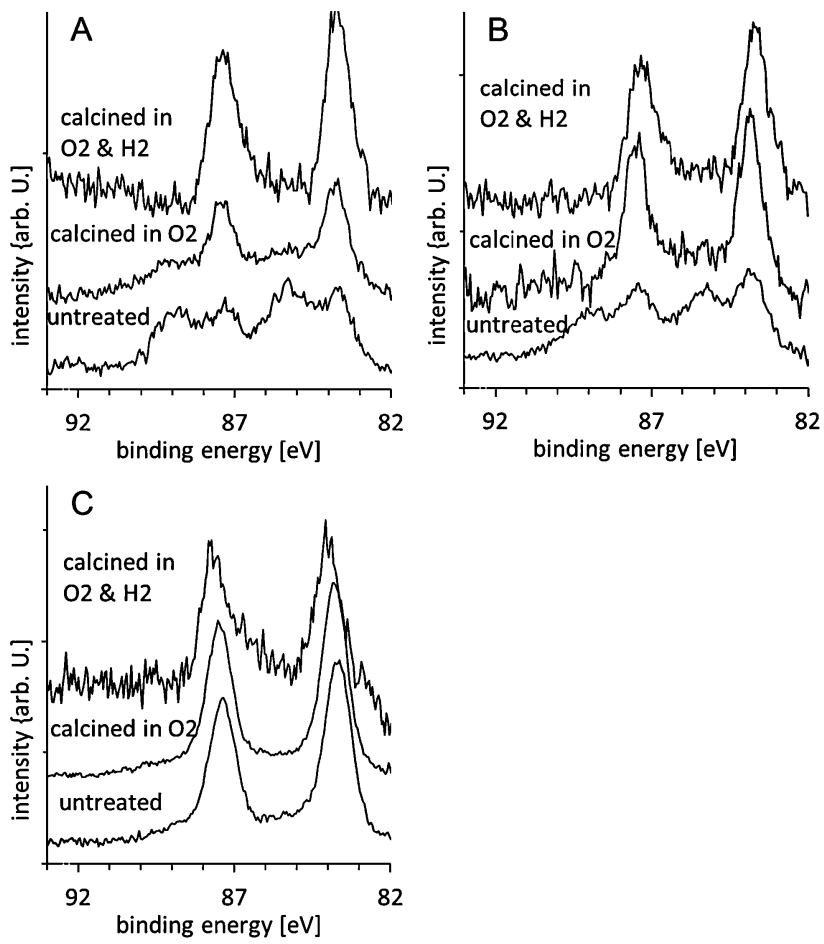

Fig. $1 \mathrm{Au}$ XPS spectra of gold clusters supported on anatase nanoparticles untreated, calcined in $\mathrm{O}_{2}$ at $200{ }^{\circ} \mathrm{C}$, and calcined in $\mathrm{O}_{2}$ and subsequently in $\mathrm{H}_{2}$ at $200{ }^{\circ} \mathrm{C}$ : (A) $\mathrm{Au}_{8}$ (B) $\mathrm{Au}_{9}$ and, (C) $\mathrm{Au}_{101}$. The spectra are normalised such that the total $\mathrm{Au}$ intensity is the same for all spectra.

This difference highlights the importance of surface pre-treatment in controlling aggregation of these clusters during deposition.
The $\mathrm{P} 2 \mathrm{p}$ spectra for the $\mathrm{Au}_{8}, \mathrm{Au}_{9}$, and $\mathrm{Au}_{101}$ clusters of the untreated samples are shown in the lower traces of Fig. 3A-C. The spectra are fitted with two peaks, one at $131.9 \pm 0.2 \mathrm{eV}$, which we refer to as the phosphorous low binding energy peak (P-LBP), and another at $133.1 \pm 0.2 \mathrm{eV}$, which we refer to as the high binding energy peak (P-HBP). The P-LBP is assigned to phosphine ligands bonded to the gold cluster core, whereas the $\mathrm{P}-\mathrm{HBP}$ is assigned to a phosphorous oxide-like chemical species formed by phosphine ligands dislodging from the cluster metal core and oxidising by interaction with the oxide support. ${ }^{16}$ Fig. 3 and 4A show that for all clusters the P-HBP is the dominant peak. This is quantified in Table 1 , which shows that the ratio of both Au-HBP/P-LBP and total-Au/P-LBP is much larger than the stoichiometric ratio of $\mathrm{Au} / \mathrm{P}$ in the pristine clusters $\left(\mathrm{Au} / \mathrm{P}=1: 1\right.$ in $\mathrm{Au}_{8}, 1.1: 1$ in $\mathrm{Au}_{9}$ and $5: 1$ in $\left.\mathrm{Au}_{101}\right)$ while the $\mathrm{P}$ intensity is mainly found in the $\mathrm{P}-\mathrm{HBP}$. In contrast, the ratio of total-Au/total-P is smaller than the stoichiometric ratio $\mathrm{Au} / \mathrm{P}$ in the pristine clusters. Dislodging the phosphine ligands from the gold cluster cores yet keeping these dislodged ligands on the titania surface to form partially oxidised species can explain the high Au-HBP/P-LBP and total-Au/P-LBP ratios. The large amount of phosphorous oxide-like species can be explained by strong binding of the phosphorous to the oxygen of the metal oxide surface. The small $\mathrm{Au} /$ total $\mathrm{P}$ ratio shows that the gold clusters agglomerate and form larger particles. Due to the limited electron mean free path of the emitted electrons, the total gold intensity for larger particles will be lower than that of small clusters given the same total gold loading on the surface. These findings are very different to our previous work
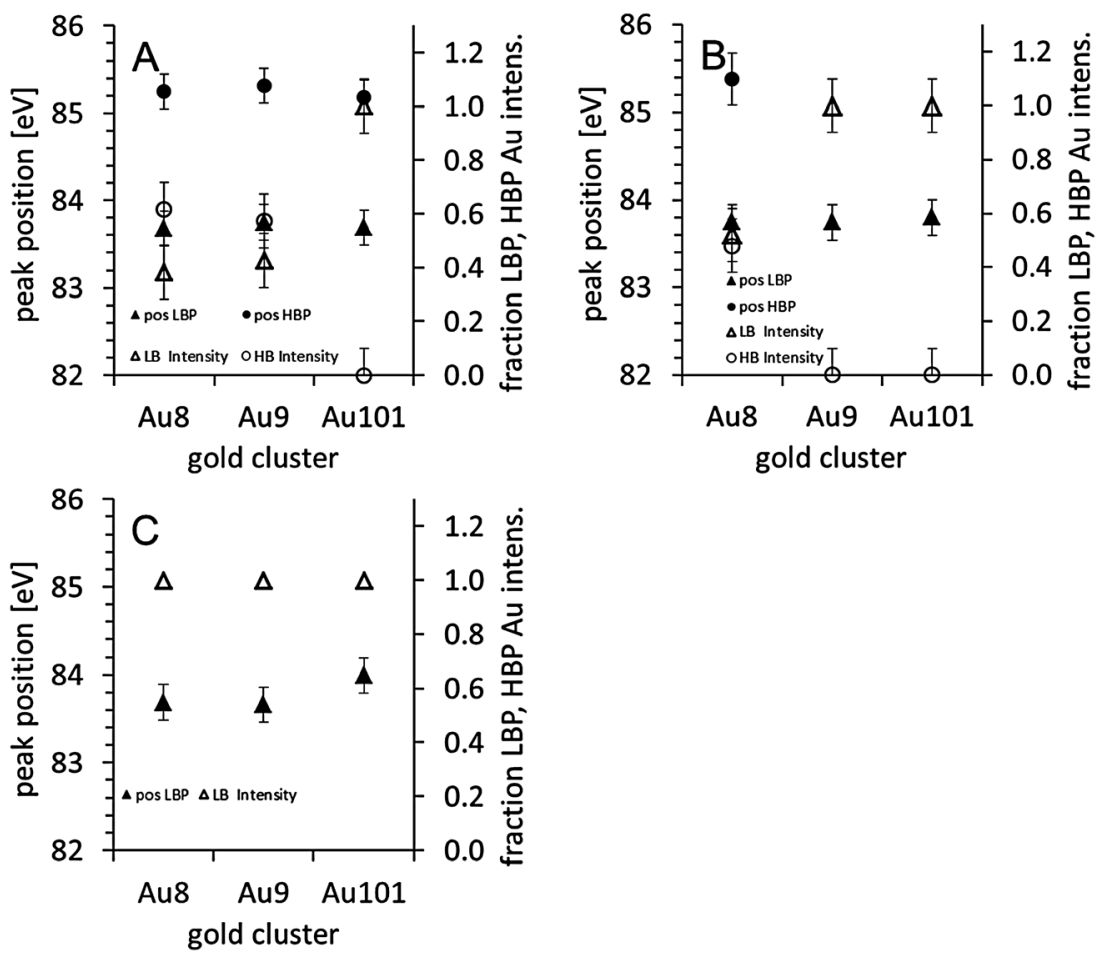

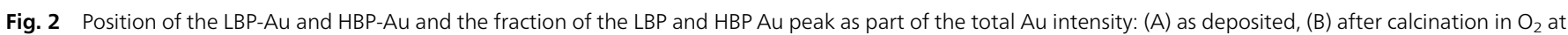
$200{ }^{\circ} \mathrm{C}$, and (C) after calcination in $\mathrm{O}_{2}$ and subsequently in $\mathrm{H}_{2}$ at $200{ }^{\circ} \mathrm{C}$. 
Table 1 Position and FWHM of the Au $4 \mathrm{f}$ binding energy peaks for each of the untreated and treated samples

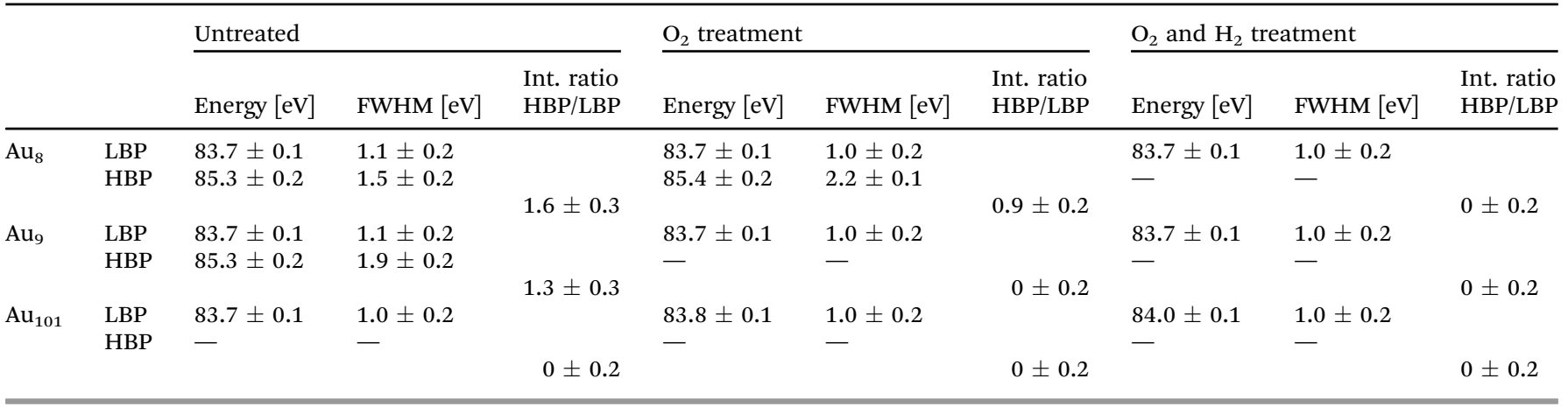
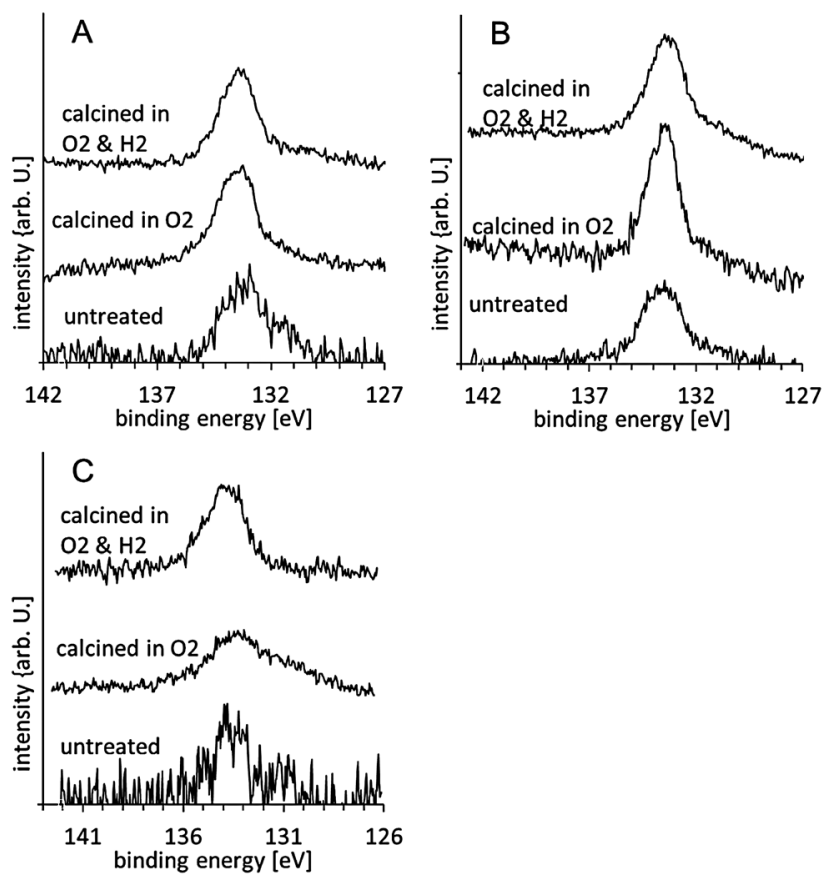

Fig. 3 P XPS spectra of gold clusters supported on anatase nanoparticles untreated, in $\mathrm{O}_{2}$ at $200{ }^{\circ} \mathrm{C}$, and calcined in $\mathrm{O}_{2}$ and subsequently in $\mathrm{H}_{2}$ at $200{ }^{\circ} \mathrm{C}:(\mathrm{A}) \mathrm{Au}_{8},(\mathrm{~B}) \mathrm{Au}_{9}$ and, (C) $\mathrm{Au}_{101}$. The spectra are normalised such that the total $\mathrm{P}$ intensity is the same for all spectra.

when the clusters are deposited on acid pre-treated P-25 Aeroxide titania, where the majority of the phosphorous intensity was found in the P-LBP. ${ }^{16}$ Acidic pretreatment changes the termination of the surface and thus influences the interaction of the clusters with the surface which is the likely reason for the difference in agglomeration of the clusters between our present and the previous work.

In summary, the deposition of $\mathrm{Au}_{8}, \mathrm{Au}_{9}$ and $\mathrm{Au}_{101}$ on the untreated anatase nanoparticles results in the removal of some triphenylphosphine ligands. A considerable fraction of the $\mathrm{Au}_{8}$ and $\mathrm{Au}_{9}$ clusters maintain their size and the remainder undergo some form of partial agglomeration. The XPS results do not permit conclusions to be drawn about aggregation of $\mathrm{Au}_{101}$, since the binding energy position of the pristine clusters is already close to the value for bulk gold.
Samples calcined in $\mathrm{O}_{2}$ at $200{ }^{\circ} \mathrm{C}$. The Au $4 \mathrm{f}$ spectra for the $\mathrm{Au}_{8}, \mathrm{Au}_{9}$, and $\mathrm{Au}_{101}$ clusters deposited on anatase particles and calcined under $\mathrm{O}_{2}$ atmosphere are shown in the middle traces of Fig. 1A-C. The spectrum of $\mathrm{Au}_{8}$ shows two doublets whereas the $\mathrm{Au}_{9}$ and $\mathrm{Au}_{101}$ spectra show only one (although there is a negligibly small second doublet for $\mathrm{Au}_{101}$ ). For $\mathrm{Au}_{8}$, the position of the Au-LBP is at $83.7 \pm 0.1 \mathrm{eV}$ and that of the Au-HBP peak at $85.4 \pm 0.2 \mathrm{eV}$, as listed in Table 1 (the fit for the $\mathrm{Au}_{8}$ spectrum is shown in the ESI $\dagger$ ). For $\mathrm{Au}_{8}$, the peak areas of Au-HBP and $\mathrm{Au}-\mathrm{LBP}$ are almost the same while for $\mathrm{Au}_{9}$ and $\mathrm{Au}_{101}$ the signal intensity is almost exclusively in the Au-LBP, as can be seen in Fig. 2B. The FWHM of the Au-LBP is also considerably smaller than that of the Au-HBP, as observed for the untreated samples.

The $\mathrm{P} 2 \mathrm{p}$ spectra for the $\mathrm{Au}_{8}, \mathrm{Au}_{9}$, and $\mathrm{Au}_{101}$ clusters are shown in the middle traces of Fig. 3A-C. The spectra can be mostly fitted with a single P-HBP but the $\mathrm{Au}_{8}$ and $\mathrm{Au}_{101}$ spectra require a small component of P-LBP to fit the spectrum albeit with large uncertainty (see Fig. 4B and Table 2). Table 2 shows similar ratios of $\mathrm{Au}-\mathrm{HBP} / \mathrm{P}-\mathrm{LBP}$ and total-Au/P-LBP for $\mathrm{Au}_{8}$ relative to the untreated clusters (also with large uncertainty). Table 2 also shows that the ratio of total-Au/ total-P is smaller than the stoichiometric ratio of $\mathrm{Au} / \mathrm{P}$ in the pristine $\mathrm{Au}_{8}$ clusters. It is not possible to draw any conclusions about $\mathrm{Au}_{101}$ since the binding energy does not change upon treatment.

The interpretation of these findings is similar to that observed for the untreated samples. The phosphorous ligands are removed from the clusters and deposited on the titania surface while the gold cluster cores either partially or fully agglomerate. The only difference is that the removal and oxidation of ligands, and the agglomeration of the cluster cores has progressed further compared to the untreated samples. In comparison to our earlier study using clusters deposited onto acid pre-washed P-25 titania, we observe more pronounced cluster core aggregation in the case of clusters deposited on untreated anatase titania and heat-treated (at the same temperature and duration) under $\mathrm{O}_{2}$ atmosphere. This shows that the acidic pretreatment not only influences the deposition of the pristine clusters but also the agglomeration of the clusters during heat treatment. Acidic pretreatment changes the termination of the surface and thus influences the interaction of the 

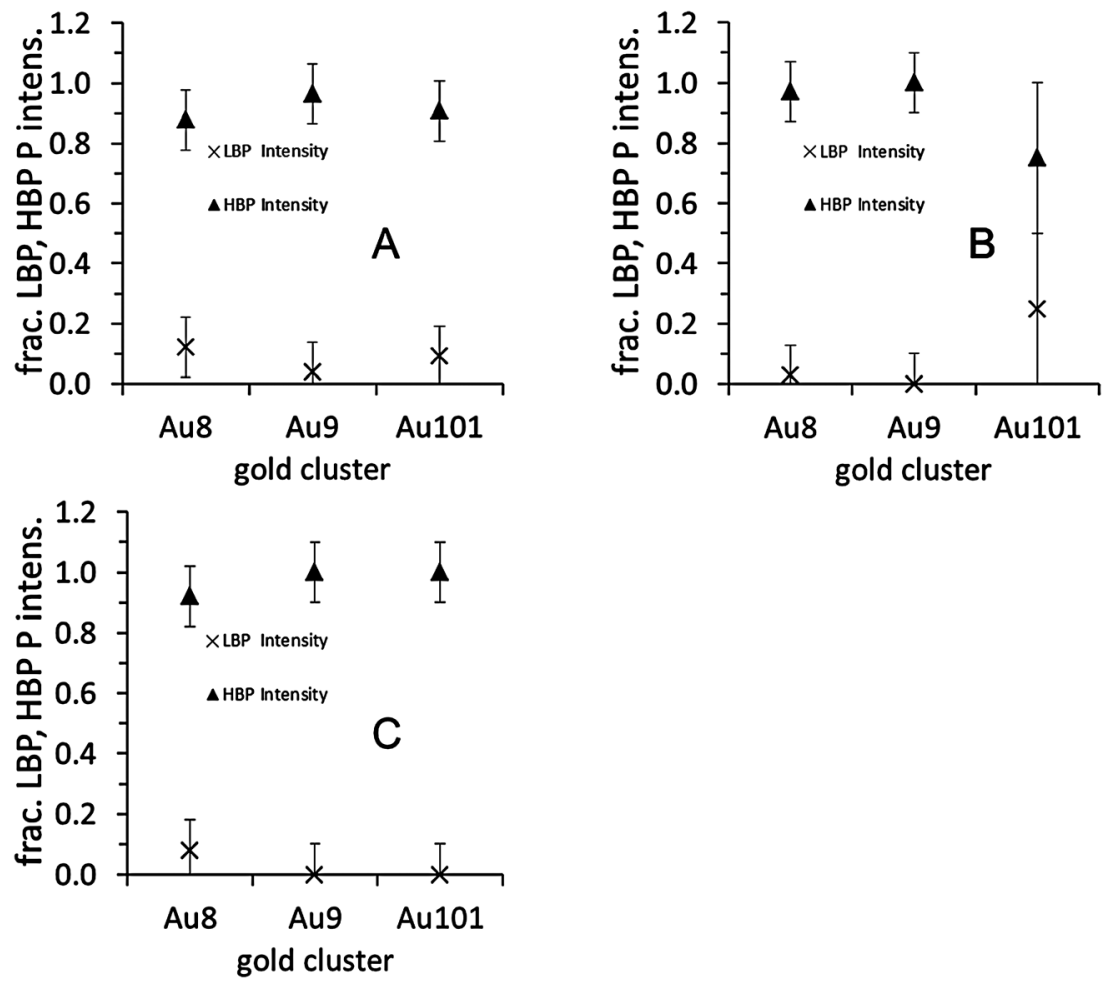

Fig. 4 Fraction of the LBP and HBP phosphorous intensity: (A) as deposited, (B) after calcination in $\mathrm{O}_{2}$ at $200{ }^{\circ} \mathrm{C}$ ( (C) after calcination in $\mathrm{O}_{2}$ and subsequently in $\mathrm{H}_{2}$ at $200^{\circ} \mathrm{C}$

Table 2 Intensity ratios between Au and P binding energy peaks for each of the untreated and treated samples

\begin{tabular}{|c|c|c|c|}
\hline & $\mathrm{Au}-\mathrm{HBP} / \mathrm{P}-\mathrm{LBP}$ & Total Au/P-LBP & $\begin{array}{l}\text { Total } \\
\text { Au/total P }\end{array}$ \\
\hline $\mathrm{Au}_{8}$ untreated & $2.2 \pm 0.5$ & $3.6 \pm 0.5$ & $0.4 \pm 0.1$ \\
\hline $\mathrm{Au}_{9}$ untreated & $5.7 \pm 1$ & $8.6 \pm 1$ & $0.5 \pm 0.1$ \\
\hline $\mathrm{Au}_{101}$ untreated & $5.2 \pm 1$ & $27.3 \pm 3$ & $2.5 \pm 0.5$ \\
\hline $\mathrm{Au}_{8}$ calcined $\mathrm{O}_{2}$ & $2.6 \pm 2$ & $5.4 \pm 4$ & $0.2 \pm 0.1$ \\
\hline $\mathrm{Au}_{9}$ calcined $\mathrm{O}_{2}$ & $\infty^{a}$ & $\infty^{a}$ & $0.1 \pm 0.1$ \\
\hline $\mathrm{Au}_{101}$ calcined $\mathrm{O}_{2}$ & $0.3 \pm 0.3$ & $1.9 \pm 2$ & $0.5 \pm 0.2$ \\
\hline $\mathrm{Au}_{8}$ calcined $\mathrm{O}_{2}$ and $\mathrm{H}_{2}$ & $0 \pm 0.2$ & $0.9 \pm 1$ & $0.1 \pm 0.1$ \\
\hline $\mathrm{Au}_{9}$ calcined $\mathrm{O}_{2}$ and $\mathrm{H}_{2}$ & $\infty^{a}$ & $\infty^{a}$ & $0.1 \pm 0.1$ \\
\hline $\mathrm{Au}_{101}$ calcined $\mathrm{O}_{2}$ and $\mathrm{H}_{2}$ & $\infty^{a}$ & $\infty^{a}$ & $0.1 \pm 0.1$ \\
\hline
\end{tabular}

clusters with the surface which is the likely reason for the difference in agglomeration of the clusters between our present and the previous work.

In summary, the deposition of $\mathrm{Au}_{8}, \mathrm{Au}_{9}$ and $\mathrm{Au}_{101}$ on the untreated anatase nanoparticles results in the almost complete removal of the ligands after calcination in $\mathrm{O}_{2}$. Maintaining the size of the pristine cluster core is successful for a considerable fraction of the $\mathrm{Au}_{8}$ clusters, but not for $\mathrm{Au}_{9}$. This finding is difficult to rationalise given the similarity of these clusters (i.e. similar metal cluster cores, both protected by triphenylphosphine ligands and with similar nitrate counter-ions) and we are undertaking other experiments, as well as detailed modelling studies, to identify any possible reasons for the observed difference.
Samples calcined in $\mathrm{O}_{2}$ and $\mathrm{H}_{2}$ at $200{ }^{\circ} \mathrm{C}$. The $\mathrm{Au}$ 4f spectra for the $\mathrm{Au}_{8}, \mathrm{Au}_{9}$, and $\mathrm{Au}_{101}$ clusters deposited on anatase nanoparticles and calcined firstly under $\mathrm{O}_{2}$ and subsequently under $\mathrm{H}_{2}$ conditions are shown in the top traces of Fig. 1A-C. The spectra of all three samples can be fitted with a single doublet at the Au-LBP position of 83.7 to $84.0 \mathrm{eV}$, as shown in Fig. $2 \mathrm{C}$ and also Table 1. As before, the Au-LBP is assigned to agglomerated clusters. ${ }^{16}$

The P 2p spectra for the $\mathrm{Au}_{8}, \mathrm{Au}_{9}$, and $\mathrm{Au}_{101}$ clusters are shown in the top traces of Fig. 3A-C. The spectra can be fitted mostly with a single P-HBP (see Fig. 4C) but a small peak at the $\mathrm{P}$-LBP is required to fit the $\mathrm{Au}_{8}$ spectrum, again with large uncertainty (see Table 2). Table 1 shows that the Au-HBP/P-LBP and total-Au/P-LBP ratios for $\mathrm{Au}_{8}$ have decreased significantly (with large uncertainty). Table 2 also shows that the ratio of total-Au/total-P is much smaller than the stoichiometric ratio of $\mathrm{Au} / \mathrm{P}$ in the pristine clusters and slightly less than the samples calcined in only $\mathrm{O}_{2}$. The combined interpretation of these results for the case of calcination in $\mathrm{O}_{2}$ and $\mathrm{H}_{2}$, is that the phosphorous ligands are now completely detached from the gold and re-deposited onto the titania surface while the gold cluster cores fully agglomerate. The more pronounced aggregation of gold cluster cores, even for $\mathrm{Au}_{8}$, during this treatment is rationalised as being due to the extended period of exposure (twice as long) at elevated temperature, with the $\mathrm{H}_{2}$ atmosphere also possibly contributing to increased aggregation.

In summary, the deposition of $\mathrm{Au}_{8}$ and $\mathrm{Au}_{9}$ on the untreated anatase nanoparticles results in the almost complete removal 


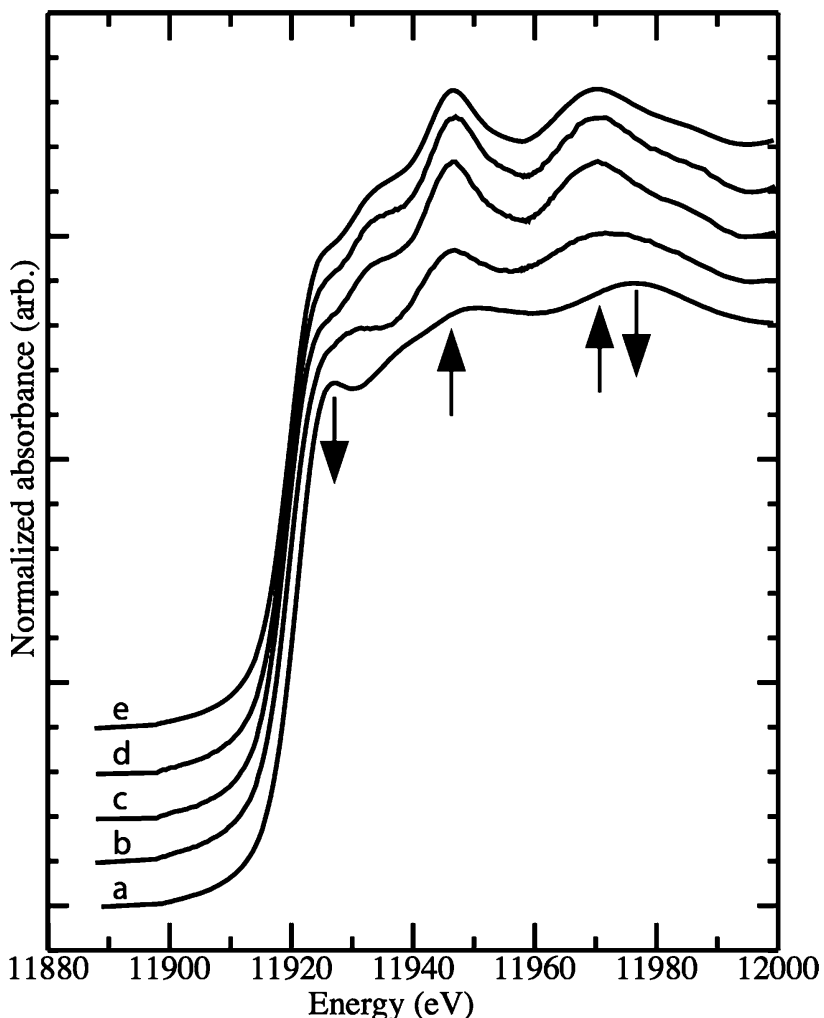

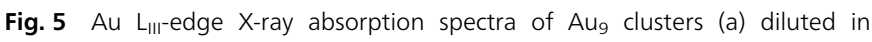
cellulose, (b) supported on anatase nanoparticles - untreated, (c) after calcination in $\mathrm{O}_{2}$ at $200{ }^{\circ} \mathrm{C}$, (d) after calcination in $\mathrm{O}_{2}$ and subsequently in $\mathrm{H}_{2}$ at $200{ }^{\circ} \mathrm{C}$, (e) bulk metal standard. The arrows indicate features that are observed to change as the samples progress from clusters to agglomerated species.

of the ligands from gold metal cores after calcination in $\mathrm{O}_{2}$ and subsequently in $\mathrm{H}_{2}$. However, the size of the gold cluster core for $\mathrm{Au}_{8}$ and $\mathrm{Au}_{9}$ is not maintained in this case but no conclusions can be drawn for $\mathrm{Au}_{101}$.

\section{B. XAS results}

Fig. 5 shows $\mathrm{Au} \mathrm{L}_{\mathrm{III}}$-edge XANES spectra for unsupported and supported $\mathrm{Au}_{9}$ clusters, bulk gold metal, as well as for $\mathrm{Au}_{9}$ clusters supported on anatase and calcined in $\mathrm{O}_{2}$ alone or $\mathrm{O}_{2}$ followed by $\mathrm{H}_{2}$. It is evident by inspection that a significant modulation of the spectrum occurs when the clusters are deposited on the anatase substrate with the peak at $\sim 11925 \mathrm{eV}$ diminishing, while broader peaks at $\sim 11947 \mathrm{eV}$ and $\sim 11970 \mathrm{eV}$ become more intense. These effects are magnified when the anatase supported clusters are calcined under either set of conditions reported above, and the XANES spectra from these two samples are visually identical to each other and to the spectrum of bulk gold. A linear regression fit of the spectra of the calcined anatase-supported clusters with a simple combination of the bulk metal and pure $\mathrm{Au}_{9}$ cluster spectra (fitted over the range 11 880-11980 eV) reinforces the result that these samples contain agglomerated gold that is chemically equivalent to the bulk gold standard (see Fig. S2 and S3 (ESI $\dagger$ ) for comparisons of these spectra). A similar fit to the anatasedeposited $\mathrm{Au}_{9}$ cluster spectrum yields a best fit of $61( \pm 3) \%$ pure
$\mathrm{Au}_{9}$ and $39( \pm 3)$ bulk gold (a comparison of the data and the fit, including a residual, is shown in Fig. S4, ESI $\dagger$ ).

The interpretations from the XAS results for the $\mathrm{Au}_{9}$ clusters are in agreement with those from the XPS data described above, i.e. that deposition of the clusters on anatase results in partial loss of phosphine ligands and concomitant agglomeration, and that subsequent calcination results in complete loss of phosphine ligands and agglomeration. This validates the conclusions from XPS for the $\mathrm{Au}_{9}$ clusters, and by extension, the conclusions for the $\mathrm{Au}_{8}$ clusters for which XAS data is not available.

\section{Conclusions}

Synchrotron-XPS measurements show that the deposition of phosphine-protected $\mathrm{Au}_{8}, \mathrm{Au}_{9}$ and $\mathrm{Au}_{101}$ clusters on untreated titania (anatase) leads to the partial removal of the ligands and their subsequent binding and oxidation to the metal oxide substrate surface. XAS data confirms these results for the $\mathrm{Au}_{9}$ clusters. The removal of ligands and the agglomeration of gold cluster cores without treatment is different to that observed by us previously, ${ }^{16}$ when the titania substrate was pre-treated with acid.

\section{Acknowledgements}

This work was supported by grants of the Australian Synchrotron and NZ Synchrotron Group (AS121/SXR/4641a\&b and AS121/ANBF/ 4438), the MacDiarmid Institute and the College of Science at the University of Canterbury, NZ. The XPS measurements were undertaken on the soft X-ray beamline at the Australian Synchrotron, Victoria, Australia. XAS data collection was undertaken at the Australian National Beamline Facility at the Photon Factory in Japan, operated by the Australian Synchrotron. We acknowledge the Australian Research Council for financial support and the High Energy Accelerator Research Organisation (KEK) in Tsukuba, Japan, for operations support.

\section{References}

1 M. Haruta, S. Tsubota, T. Kobayashi, H. Kageyama, M. J. Genet and B. Delmon, J. Catal., 1993, 144, 175-192.

2 R. Meyer, C. Lemire, S. Shaikhutdinov and H. Freund, Gold Bull., 2004, 37, 72-124.

3 A. A. Herzing, C. J. Kiely, A. F. Carley, P. Landon and G. J. Hutchings, Science, 2008, 321, 1331-1335.

4 M. Valden, X. Lai and D. W. Goodman, Science, 1998, 281, 1647-1650.

5 M. Turner, V. B. Golovko, O. P. H. Vaughan, P. Abdulkin, A. Berenguer-Murcia, M. S. Tikhov, B. F. G. Johnson and R. M. Lambert, Nature, 2008, 454, 981-983.

6 G. E. Johnson, C. Wang, T. Priest and J. Laskin, Anal. Chem., 2011, 83, 8069-8072.

7 M. Büttner and P. Oelhafen, Surf. Sci., 2006, 600, 1170-1177. 8 Y. Kitsudo, A. Iwamoto, H. Matsumoto, K. Mitsuhara, T. Nishimura, M. Takizawa, T. Akita, Y. Maeda and Y. Kido, Surf. Sci., 2009, 603, 2108-2114. 
9 D.-C. Lim, C.-C. Hwang, G. Gantefor and Y. D. Kim, Phys. Chem. Chem. Phys., 2010, 12, 15172-15180.

10 L. Benz, X. Tong, P. Kemper, H. Metiu, M. T. Bowers and S. K. Buratto, J. Phys. Chem. B, 2005, 110, 663-666.

11 S. Lee, C. Fan, T. Wu and S. L. Anderson, J. Am. Chem. Soc., 2004, 126, 5682-5683.

12 Y. Lei, F. Mehmood, S. Lee, J. Greeley, B. Lee, S. Seifert, R. E. Winans, J. W. Elam, R. J. Meyer, P. C. Redfern, D. Teschner, R. Schlögl, M. J. Pellin, L. A. Curtiss and S. Vajda, Science, 2010, 328, 224-228.

13 U. Heiz and W.-D. Schneider, Crit. Rev. Solid State Mater. Sci., 2001, 26, 251-290.

14 S. Kunz, K. Hartl, M. Nesselberger, F. F. Schweinberger, G. Kwon, M. Hanzlik, K. J. J. Mayrhofer, U. Heiz and M. Arenz, Phys. Chem. Chem. Phys., 2010, 12, 10288-10291.

15 B. Yoon, H. Häkkinen, U. Landman, A. S. Wörz, J.-M. Antonietti, S. Abbet, K. Judai and U. Heiz, Science, 2005, 307, 403-407.

16 D. P. Anderson, J. F. Alvino, A. Gentleman, H. A. Qahtani, L. Thomsen, M. I. J. Polson, G. F. Metha, V. B. Golovko and G. G. Andersson, Phys. Chem. Chem. Phys., 2013, 15, 3917-3929.

17 C. C. Chusuei, X. Lai, K. A. Davis, E. K. Bowers, J. P. Fackler and D. W. Goodman, Langmuir, 2001, 17, 4113-4117.

18 A. B. Hungria, R. Raja, R. D. Adams, B. Captain, J. M. Thomas, P. A. Midgley, V. Golovko and B. F. G. Johnson, Angew. Chem., Int. Ed., 2006, 45, 4782-4785.

19 R. Raja, V. B. Golovko, J. M. Thomas, A. Berenguer-Murcia, W. Zhou, S. Xie and B. F. G. Johnson, Chem. Commun., 2005, 2026-2028.

20 R. Zanella and C. Louis, Catal. Today, 2005, 107-108, 768-777.

21 Y. Yuan, K. Asakura, H. Wan, K. Tsai and Y. Iwasawa, Chem. Lett., 1996, 755.

22 H. G. Boyen, A. Ethirajan, G. Kästle, F. Weigl, P. Ziemann, G. Schmid, M. G. Garnier, M. Büttner and P. Oelhafen, Phys. Rev. Lett., 2005, 94, 016804.

23 H. Yin, Z. Ma, M. Chi and S. Dai, Catal. Lett., 2010, 136, 209-221.
24 C. G. Long, J. D. Gilbertson, G. Vijayaraghavan, K. J. Stevenson, C. J. Pursell and B. D. Chandler, J. Am. Chem. Soc., 2008, 130, 10103-10115.

25 V. Borman, M. Pushkin, V. Tronin and V. Troyan, J. Exp. Theor. Phys., 2010, 110, 1005-1025.

26 C. Battistoni, G. Mattogno, F. Cariati, L. Naldini and A. Sgamellotti, Inorg. Chim. Acta, 1977, 24, 207-210.

27 D. M. Cox, W. Eberhardt, P. Fayet, Z. Fu, B. Kessler, R. D. Sherwood, D. Sondericker and A. Kaldor, Z. Phys. D: At., Mol. Clusters, 1991, 20, 385-386.

28 S. B. DiCenzo, S. D. Berry and E. H. Hartford, Jr., Phys. Rev. B: Condens. Matter Mater. Phys., 1988, 38, 8465-8468.

29 Y. Lykhach, J. Plšek, I. Spirovová and Z. Bastl, Collect. Czech. Chem. Commun., 2003, 68, 1791-1804.

30 Y. Yuan, K. Asakura, A. P. Kozlova, H. Wan, K. Tsai and Y. Iwasawa, Catal. Today, 1998, 44, 333-342.

31 Y. Zhu, H. Qian and R. Jin, Chem.-Eur. J., 2010, 16, 11455-11462. 32 Y.-Y. Fong, B. R. Visser, J. R. Gascooke, B. C. C. Cowie, L. Thomsen, G. F. Metha, M. A. Buntine and H. H. Harris, Langmuir, 2011, 27, 8099-8104.

33 B. C. C. Cowie, A. Tadich and L. Thomsen, AIP Conf. Proc., 2010, 1234, 307-310.

34 D. A. Shirley, Phys. Rev. B: Condens. Matter Mater. Phys., 1972, 5, 4709-4714.

35 J. Végh, J. Electron Spectrosc. Relat. Phenom., 2006, 151, 159-164. 36 H. P. Hughes and J. A. Scarfe, J. Phys.: Condens. Matter, 1996, 8, 1421.

37 J. F. Moulder, W. F. Stickle, P. E. Sobol and K. D. Bomben, Handbook of X-ray Photoelectron Spectroscopy, Physical Electronics, Inc., Eden Prairie, 1995.

38 J. J. Yeh and I. Lindau, At. Data Nucl. Data Tables, 1985, 32, 1-155.

39 R. I. Derby, W. S. Hutchinson, T. H. Dexter and J. M. Naughton, Inorganic Syntheses, John Wiley \& Sons, Inc., 2007, pp. 152-158.

40 W. W. Weare, S. M. Reed, M. G. Warner and J. E. Hutchison, J. Am. Chem. Soc., 2000, 122, 12890-12891.

41 D. Briggs and M. P. Seah, Practical surface analysis, Wiley, Chichester, New York, Aarau, 1990. 